\title{
Inverse finite element modelling of shells using the degenerate solid approach
}

\author{
Víctor D. Fachinottia,*, Alejandro E. Albanesi ${ }^{\mathrm{a}}$, José M. Martinez Valle ${ }^{\mathrm{b}}$ \\ ${ }^{a}$ Centro de Investigación de Métodos Computacionales(CIMEC), Universidad Nacional \\ del Litoral (UNL)/ Consejo Nacional de Investigaciones Científicas y Técnicas \\ (CONICET), Predio CCT-CONICET Santa Fe, Colectora Ruta Nac. 168, Paraje El \\ Pozo, 3000, Santa Fe, Argentina \\ ${ }^{b}$ Departamento de Mecánica, Escuela Politécnica Superior, Universidad de Córdoba, \\ Edificio Leonardo da Vinci, Campus de Rabanales, 14071, Córdoba, España
}

\section{Abstract}

The inverse finite element method (IFEM) for degenerate solid shells is introduced. IFEM allows determining the undeformed shape of a body (in this case, a shell-like body) such that it attains a desired shape after large elastic deformations. The model is based on the degenerate solid approach, which enables the use of the standard constitutive laws of Solid Mechanics. First, IFEM is applied to three popular benchmarks for validation purposes. Then, the capabilities of IFEM for inverse design are demonstrated by means of its application to the design of a microvalve.

Keywords: Inverse Finite Element Method; degenerate solid shells; Mixed Interpolation of Tensorial Components; large elastic deformations.

\footnotetext{
${ }^{*}$ Corresponding author

Email address: vfachino@intec.unl.edu.ar (Víctor D. Fachinotti )
} 


\section{Introduction}

The Inverse Finite Element Method (IFEM) is the Finite Element Method (FEM) applied to the problem of determining the undeformed configuration of a body when the deformed configuration as well as the actuating loads are known. This kind of problem -also known as the Inverse Design problem- often arises in the design of compliant structures or mechanisms suffering large elastic displacements and/or rotations, for instance: a gasket that deforms to the desired shape under given loads [1]; a rubber seal that closes a given channel under a given pressure [2]; a turbine blade that attains an optimal shape at a certain angular speed [3]; an S-clutch whose shoes exactly engage the friction surface of a given drum at a given angular speed $[4,5]$; a device that folds an intraocular lens in such a way that facilitates its implantation into the eye [6], among other interesting applications developed in the papers mentioned.

Outside the field of inverse design, Lu and Zhou [7, 8] proposed an application of IFEM to the prevention of aneurysms, taking the in vivo image of an aneurysm as the known deformed configuration under a known pressure.

All these inverse problems could be solved using systematized "trial-anderror" methods from Optimization Theory, considering any measure of the closeness to the desired deformed configuration as the cost function to be minimized. At each iteration of the optimization problem, a nonlinear (direct) equilibrium equation has to be solved to determine the cost function. In contrast, IFEM solves only one nonlinear equilibrium equation to determine the desired deformed configuration, which is approximately as computationally expensive as only one iteration of an optimization problem. This was 
illustrated by Albanesi et al. [4, 5], who used IFEM to design a compliant gripper, which had been originally designed by Lan and Cheng [9] by solving an optimization problem.

In our previous papers, IFEM was introduced for 3D solids [3] and 3D beams $[4,5]$. The current paper is a step towards the completion of our IFEM library by introducing shell elements.

Zhou and Lu [8] introduced IFEM for shells using the stress-resultant approach proposed by Simo et al. [10]. Models based in this approach need specialized constitutive equations for the accross-the-thickness membrane and shear stress resultants and stress couple, as described in the pioneering work of Simo and Fox [11].

In the present paper, the degenerate solid approach to shells, originally proposed by Ahmad et al. [12] and extended to nonlinear geometrical analysis by Ramm [13], is preferred. This approach is characterized by defining the stress itself (rather than the stress resultants) using the same constitutive equations as those of Solid Mechanics. This attribute of the degenerate solid shells was the reason for our choice. Then, as an original contribution, we introduce IFEM to the context of degenerate solid shells.

The low-order displacement-based shell finite elements predict spurious shear stresses and, as result, exhibit artificially high stiffness. This is the well-known "shear locking" defect [14], which can be circumvented by using the appropriate mixed finite elements. In the present paper, recourse is made to the formulation known as Mixed Interpolation of Tensorial Components (MITC), originally proposed by Dvorkin and Bathe [15] for bi-linear 4-node quadrangles and extended by Bucalem and Bathe [16] to bi-quadratic 9- 
node and bi-cubic 16-node quadrangles. Using MITC, the components of the strain tensor are interpolated independently of the displacements, in order to preclude shear locking.

First, we solve three popular problems for linear-elastic shells with large deflections and rotations [17], using these benchmarks for the purpose of validating the presented IFEM. Finally, the capability of IFEM for inverse design is shown by the design of a compliant microvalve to close a given channel when the pressure drop attains a prescribed value, giving a more efficient alternative to that originally proposed by Seidemann et al. [18].

\section{Formulation of the degenerate solid shell finite element}

The aim of this section is to give a brief summary of the formulation of FEM for degenerate solid shells, which is already classical in the "direct" FEM. Specifically, we describe the so-called "basic shell" model [19, 20], which is based on the Mindlin-Reissner kinematic hypothesis: those straight fibers that are normal to the midsurface of the shell when it is undeformed remain straight and unstretched during deformation. The "basic shell" model is well-suited for thin to moderately thick shells, offering the best compromise between simplicity and applicability in FEM for shells.

As a corollary, we arrive at a system of discrete nonlinear equations governing the equilibrium of geometrically nonlinear degenerate solid shells in "direct" FEM, to be taken as the starting point for the development of IFEM for degerate solid shells in the next section. 


\subsection{Kinematic hypotheses for shells}

Let $\mathcal{B}^{0}$ represent the solid shell body shown in Fig. 1. The geometry of the shell is defined by its midsurface $\mathcal{S}^{0}$ and the thickness of the shell at each point of the midsurface. Let $\left\{\xi_{1}, \xi_{2}, \xi_{3}\right\}$ be a system of natural coordinates, such that $\xi_{1}$ and $\xi_{2}$ vary through the midsurface $\mathcal{S}^{0}$ and $\xi_{3}$ varies across the thickness of the shell, with $-1 \leq \xi_{i} \leq 1$ and $\xi_{3}=0$ at the midsurface. Then, the position of any point $\boldsymbol{X} \in \mathcal{B}^{0}$ can be expressed as a function of the natural coordinates as follows:

$$
\boldsymbol{X}\left(\xi_{1}, \xi_{2}, \xi_{3}\right)=\overline{\boldsymbol{X}}\left(\xi_{1}, \xi_{2}\right)+\xi_{3} \frac{H}{2} \boldsymbol{T}\left(\xi_{1}, \xi_{2}\right)
$$

where $\overline{\boldsymbol{X}} \in \mathcal{S}^{0}, \boldsymbol{T}$ is the unit vector known as the material director, and $H=H\left(\xi_{1}, \xi_{2}\right)$ is the thickness of the undeformed shell.
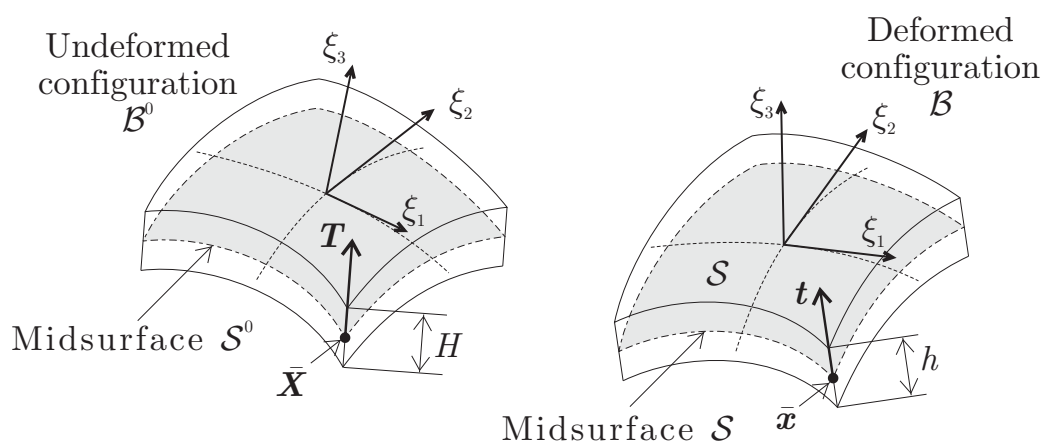

Figure 1: Geometric representation of the undeformed and deformed configurations of a shell.

Let $\mathcal{B}$ be the deformed configuration of the shell, with midsurface $\mathcal{S}$. After deformation, the point $\boldsymbol{X} \in \mathcal{B}^{0}$ occupies the position $\boldsymbol{x} \in \mathcal{B}$ :

$$
\boldsymbol{x}\left(\xi_{1}, \xi_{2}, \xi_{3}\right)=\overline{\boldsymbol{x}}\left(\xi_{1}, \xi_{2}\right)+\xi_{3} \frac{h}{2} \boldsymbol{t}\left(\xi_{1}, \xi_{2}\right)
$$


where $\overline{\boldsymbol{x}} \in \mathcal{S}, \boldsymbol{t}$ is the unit vector known as the spatial director, and $h=$ $h\left(\xi_{1}, \xi_{2}\right)$ is the thickness of the undeformed shell.

In the present paper, we adopt the "basic shell" model [19, 20], based on the Mindlin-Reissner plate theory, according to which $\boldsymbol{t}$ is not necessarily normal to $\mathcal{S}$ if $\boldsymbol{T}$ is normal to $\mathcal{S}^{0}$ (and viceversa), as an effect of the shear deformation. Further, as a consequence of the Mindlin-Reissner assumption, the strain normal to the midsurface is null [20], so that the thickness of the shell remains constant during deformation, i.e., $h=H$.

Inside a generic finite element with nodes $i=1,2, \ldots, N$, the positions $\boldsymbol{x} \in \mathcal{B}$ and $\boldsymbol{X} \in \mathcal{B}^{0}$ are isoparametrically interpolated from their respective nodal values, as follows:

$$
\begin{gathered}
\boldsymbol{X}\left(\xi_{1}, \xi_{2}, \xi_{3}\right)=\varphi_{i}\left(\xi_{1}, \xi_{2}\right)\left[\overline{\boldsymbol{X}}_{i}+\frac{\xi_{3}}{2} h\left(\xi_{1}, \xi_{2}\right) \boldsymbol{T}_{i}\right]=\boldsymbol{\Phi}\left(\xi_{1}, \xi_{2}, \xi_{3}\right) \boldsymbol{Q}, \\
\boldsymbol{x}\left(\xi_{1}, \xi_{2}, \xi_{3}\right)=\varphi_{i}\left(\xi_{1}, \xi_{2}\right)\left[\overline{\boldsymbol{x}}_{i}+\frac{\xi_{3}}{2} h\left(\xi_{1}, \xi_{2}\right) \boldsymbol{t}_{i}\right]=\boldsymbol{\Phi}\left(\xi_{1}, \xi_{2}, \xi_{3}\right) \boldsymbol{q},
\end{gathered}
$$

with

$$
\begin{aligned}
& \boldsymbol{\Phi}=\left[\begin{array}{lllll}
\varphi_{1} \boldsymbol{I}_{3 \times 3} & \frac{\xi_{3}}{2} h \varphi_{1} \boldsymbol{I}_{3 \times 3} & \cdots & \varphi_{N} \boldsymbol{I}_{3 \times 3} & \frac{\xi_{3}}{2} h \varphi_{N} \boldsymbol{I}_{3 \times 3}
\end{array}\right], \\
& \boldsymbol{Q}=\left[\begin{array}{c}
\overline{\boldsymbol{X}}_{1} \\
\boldsymbol{T}_{1} \\
\vdots \\
\overline{\boldsymbol{X}}_{N} \\
\boldsymbol{T}_{N}
\end{array}\right], \quad \boldsymbol{q}=\left[\begin{array}{c}
\overline{\boldsymbol{x}}_{1} \\
\boldsymbol{t}_{1} \\
\vdots \\
\overline{\boldsymbol{x}}_{N} \\
\boldsymbol{t}_{N}
\end{array}\right],
\end{aligned}
$$

where $\left(\overline{\boldsymbol{X}}_{i}, \boldsymbol{T}_{i}\right)$ defines the position of node $i$ in the finite element mesh representing $\mathcal{B}^{0}$ (known for FEM, unknown for IFEM), $\left(\overline{\boldsymbol{x}}_{i}, \boldsymbol{t}_{i}\right)$ defines the position of node $i$ in the mesh representing $\mathcal{B}$ (unknown for FEM, known for IFEM), 
and $\varphi_{i}=\varphi_{i}\left(\xi_{1}, \xi_{2}\right)$ is the 2-D shape function associated to node $i ; \boldsymbol{I}_{3 \times 3}$ is the $3 \times 3$ identity matrix.

The deformation of the shell can be measured using the Green-Lagrange strain tensor, which can be expressed as

$$
\boldsymbol{E}=\underbrace{\frac{1}{2}\left(\boldsymbol{g}_{\alpha} \cdot \boldsymbol{g}_{\beta}-\boldsymbol{G}_{\alpha} \cdot \boldsymbol{G}_{\beta}\right)}_{E_{\alpha \beta}^{\text {cov }}} \boldsymbol{G}^{\alpha} \otimes \boldsymbol{G}^{\beta},
$$

where $E_{\alpha \beta}^{\text {cov }}$ are the so-called covariant components of $\boldsymbol{E}, \boldsymbol{g}_{\alpha}=\partial \boldsymbol{x} / \partial \xi_{\alpha}$ and $\boldsymbol{G}_{\alpha}=\partial \boldsymbol{X} / \partial \xi_{\alpha}$ are the spatial and convective basis vectors, respectively, and $\boldsymbol{G}^{\alpha}$ is a vector of the base reciprocal to $\left\{\boldsymbol{G}_{\alpha}\right\}$, so that $\boldsymbol{G}^{\alpha} \cdot \boldsymbol{G}_{\beta}=\delta_{\beta}^{\alpha}$.

Using FEM, the covariant strain components $E_{\alpha \beta}^{\mathrm{cov}}$ take the form

$$
E_{\alpha \beta}^{\mathrm{cov}}=\frac{1}{2}\left(\boldsymbol{q}^{T} \boldsymbol{A}_{\alpha \beta} \boldsymbol{q}-\boldsymbol{Q}^{T} \boldsymbol{A}_{\alpha \beta} \boldsymbol{Q}\right)
$$

where $\boldsymbol{A}_{\alpha \beta}$ is the $6 N \times 6 N$-symmetric matrix defined by

$$
\boldsymbol{A}_{\alpha \beta}=\frac{1}{2}\left(\frac{\partial \boldsymbol{\Phi}^{T}}{\partial \xi_{\alpha}} \frac{\partial \boldsymbol{\Phi}}{\partial \xi_{\beta}}+\frac{\partial \boldsymbol{\Phi}^{T}}{\partial \xi_{\beta}} \frac{\partial \boldsymbol{\Phi}}{\partial \xi_{\alpha}}\right) .
$$

\subsection{The cure for shear locking}

The stiffness of low-order finite elements increases spuriously as the thickness/in-plane dimension of the element decreases. This is the well-known "shear locking" problem, which affects even cubic order elements.

One of the simpler cures for "shear locking" is the use of the "assumedstrain" technique. In particular, we use the MITC formulation, originally proposed by Dvorkin and Bathe [15].

Using MITC, each covariant strain field $E_{\alpha \beta}^{\text {cov }}$ (that defined by Eq. (8)) is replaced by an "assumed" field $\tilde{E}_{\alpha \beta}^{\text {cov }}$. Inside each MITC finite element, the 
assumed field $\tilde{E}_{\alpha \beta}^{\text {cov }}$ is defined so as to coincide with $E_{\alpha \beta}^{\text {cov }}$ at a series of "tying" points:

$$
\tilde{E}_{\alpha \beta}^{\mathrm{cov}}\left(\xi_{1}^{I}, \xi_{2}^{I}, \xi_{3}^{I}\right) \equiv E_{\alpha \beta}^{\mathrm{cov}}\left(\xi_{1}^{I}, \xi_{2}^{I}, \xi_{3}^{I}\right) \quad I=1,2, \ldots, n_{\alpha \beta}
$$

where $\left(\xi_{1}^{I}, \xi_{2}^{I}, \xi_{2}^{I}\right)$ are the natural coordinates of the tying point $I$.

With quadrangular MITC $n$ elements (where $n$ stands for the number of nodes of the element, e.g., MITC4 [15], MITC9, MITC16 [16]), the assumed strain can be defined as

$$
\tilde{E}_{\alpha \beta}^{\mathrm{cov}}\left(\xi_{1}, \xi_{2}, \xi_{3}\right)=\sum_{K=1}^{n_{\alpha \beta}} \tilde{\varphi}_{\alpha \beta}^{I}\left(\xi_{1}, \xi_{2}, \xi_{3}\right) E_{\alpha \beta}\left(\xi_{1}^{I}, \xi_{2}^{I}, \xi_{3}^{I}\right)
$$

where $\tilde{\varphi}_{\alpha \beta}^{I}$ is the Lagrange polynomial associated to the tying point $I$, such that $\tilde{\varphi}_{\alpha \beta}^{I}\left(\xi_{1}^{J}, \xi_{2}^{J}, \xi_{2}^{J}\right)=\delta_{I J}$ at any tying point $J$ associated to the covariant strain $E_{\alpha \beta}$.

Algorithmically, the use of MITCn elements amounts to replacing the matrix $\boldsymbol{A}_{\alpha \beta}$, Eq. (9), by

$$
\tilde{\boldsymbol{A}}_{\alpha \beta}\left(\xi_{1}, \xi_{2}, \xi_{3}\right)=\sum_{I=1}^{n_{\alpha \beta}} \tilde{\varphi}_{\alpha \beta}^{I}\left(\xi_{1}, \xi_{2}, \xi_{3}\right) \boldsymbol{A}_{\alpha \beta}\left(\xi_{1}^{I}, \xi_{2}^{I}, \xi_{3}^{I}\right),
$$

in the definition of $E_{\alpha \beta}^{\mathrm{cov}}$, Eq. (8).

From now on, "direct" strains will be replaced with "assumed" strains, and the superimposed tilde that identifies the assumed ones will be obviated in order to simplify the notation.

\subsection{Constitutive equations in shells}

One of the characteristic features of the degenerate solid shell elements is the use of the constitutive laws for continuum solids. So, for an elastic 
solid, the constitutive law can be written as a function relating $\boldsymbol{E}$ with its work-conjugate stress, the second Piola-second Piola-Kirchhoff stress tensor $\boldsymbol{S}$, i.e.,

$$
\boldsymbol{S}=\boldsymbol{S}(\boldsymbol{E}) .
$$

Further, the "basic shell" model assumes that the stress in the direction normal to the midsurface (that of $\boldsymbol{G}^{3}$ ) is zero [19]. Then, it is convenient to refer the constitutive law to a Cartesian frame $\left\{\boldsymbol{e}_{1}, \boldsymbol{e}_{2}, \boldsymbol{e}_{3}\right\}$ attached to each point $\boldsymbol{X} \in \mathcal{B}^{0}$, such that a Cartesian plane, say $\left\{\boldsymbol{e}_{1}, \boldsymbol{e}_{2}\right\}$, is always tangent to the shell, or more precisely, to the surface $\xi_{3}=$ constant. At this point, we need to refer $\boldsymbol{E}$ to this Cartesian frame:

$$
\boldsymbol{E}=\underbrace{\theta_{i}^{\alpha} \theta_{j}^{\beta} E_{\alpha \beta}}_{E_{i j}} \boldsymbol{e}_{i} \otimes \boldsymbol{e}_{j},
$$

with

$$
\theta_{i}^{\alpha}=\boldsymbol{G}^{\alpha} \cdot \boldsymbol{e}_{i} .
$$

Using Voigt notation, the local-Cartesian and the covariant components of $\boldsymbol{E}$ are related by

$$
\check{\boldsymbol{E}}=\Theta \check{\boldsymbol{E}}^{\mathrm{cov}},
$$


with

$$
\begin{aligned}
& \check{\boldsymbol{E}}=\left[\begin{array}{llllll}
E_{11} & E_{22} & E_{33} & 2 E_{12} & 2 E_{23} & 2 E_{13}
\end{array}\right]^{T}, \\
& \check{\boldsymbol{E}}^{\mathrm{cov}}=\left[\begin{array}{llllll}
E_{11}^{\mathrm{cov}} & E_{22}^{\mathrm{cov}} & E_{33}^{\mathrm{cov}} & 2 E_{12}^{\mathrm{cov}} & 2 E_{23}^{\mathrm{cov}} & 2 E_{13}^{\mathrm{cov}}
\end{array}\right]^{T}, \\
& \boldsymbol{\Theta}=\left[\begin{array}{cccccc}
\theta_{1}^{1} \theta_{1}^{1} & \theta_{1}^{2} \theta_{1}^{2} & \theta_{1}^{3} \theta_{1}^{3} & \theta_{1}^{1} \theta_{1}^{2} & \theta_{1}^{2} \theta_{1}^{3} & \theta_{1}^{3} \theta_{1}^{1} \\
\theta_{2}^{1} \theta_{2}^{1} & \theta_{2}^{2} \theta_{2}^{2} & \theta_{2}^{3} \theta_{2}^{3} & \theta_{2}^{1} \theta_{2}^{2} & \theta_{2}^{2} \theta_{2}^{3} & \theta_{2}^{3} \theta_{2}^{1} \\
\theta_{3}^{1} \theta_{3}^{1} & \theta_{3}^{2} \theta_{3}^{2} & \theta_{3}^{2} \theta_{3}^{2} & \theta_{1}^{1} \theta_{3}^{2} & \theta_{3}^{2} \theta_{3}^{3} & \theta_{3}^{3} \theta_{1}^{1} \\
2 \theta_{1}^{1} \theta_{2}^{1} & 2 \theta_{1}^{2} \theta_{2}^{2} & 2 \theta_{1}^{3} \theta_{2}^{3} & \theta_{1}^{2} \theta_{2}^{1}+\theta_{1}^{1} \theta_{2}^{2} & \theta_{1}^{3} \theta_{2}^{2}+\theta_{1}^{2} \theta_{2}^{3} & \theta_{1}^{3} \theta_{2}^{1}+\theta_{1}^{1} \theta_{2}^{3} \\
2 \theta_{2}^{1} \theta_{3}^{1} & 2 \theta_{2}^{2} \theta_{3}^{2} & 2 \theta_{2}^{3} \theta_{3}^{3} & \theta_{2}^{2} \theta_{3}^{1}+\theta_{2}^{1} \theta_{3}^{2} & \theta_{2}^{3} \theta_{3}^{2}+\theta_{2}^{2} \theta_{3}^{3} & \theta_{2}^{3} \theta_{3}^{1}+\theta_{2}^{1} \theta_{3}^{3} \\
2 \theta_{1}^{1} \theta_{3}^{1} & 2 \theta_{1}^{2} \theta_{3}^{2} & 2 \theta_{1}^{3} \theta_{3}^{3} & \theta_{1}^{2} \theta_{3}^{1}+\theta_{1}^{1} \theta_{3}^{2} & \theta_{1}^{3} \theta_{3}^{2}+\theta_{1}^{2} \theta_{3}^{3} & \theta_{1}^{3} \theta_{3}^{1}+\theta_{1}^{1} \theta_{3}^{3}
\end{array}\right] .
\end{aligned}
$$

\subsection{The principle of virtual work in degenerate solid shells}

When a shell-like body is modeled using the degenerate solid shell FEM, the equilibrium of the body is governed by the principle of virtual work (PVW) given in the standard form for 3D solids. Using the Lagrangian for-

mulation for large deformation problems together with the Green-Lagrange strain $\boldsymbol{E}$ as a measure of the deformation, the PVW for general solids takes the form

$$
\int_{\mathcal{B}^{0}} \boldsymbol{S}: \delta \boldsymbol{E} \mathrm{d} V=\mathcal{W}^{\text {ext }}(\delta \boldsymbol{u})
$$

for all kinematically admissible displacement variations $\delta \boldsymbol{u}, \delta \boldsymbol{E}$ is the GreenLagrange strain induced by $\delta \boldsymbol{u}, \boldsymbol{S}$ is the second Piola-Kirchhoff stress tensor (work-conjugate to $\boldsymbol{E}$ ), and $\mathcal{W}^{\text {ext }}$ is the work of the external forces (surface tractions and body forces) on the whole body under a displacement $\delta \boldsymbol{u}$.

In the "direct" FEM, where $\boldsymbol{X}$ and $\boldsymbol{x}$ are interpolated according to Eqs. (3) and (4) and $\boldsymbol{X}$ is known, the displacement variation can be written as

$$
\delta \boldsymbol{u}=\delta \boldsymbol{x}=\boldsymbol{\Phi} \delta \boldsymbol{q}
$$


with

$$
\delta \boldsymbol{q}=\left[\begin{array}{c}
\delta \overline{\boldsymbol{x}}_{1} \\
\delta \boldsymbol{t}_{1} \\
\vdots \\
\delta \overline{\boldsymbol{x}}_{N} \\
\delta \boldsymbol{t}_{N}
\end{array}\right]
$$

where $\delta \overline{\boldsymbol{x}}_{i}$ and $\delta \boldsymbol{t}_{i}$ denote admissible variations of $\overline{\boldsymbol{x}}$ and $\boldsymbol{t}$ at node $i$.

Under a variation $\delta \boldsymbol{q}$, the covariant strain components given by Eq. (8) undergo the following variation (written in Voigt notation):

$$
\delta \check{\boldsymbol{E}}^{\mathrm{cov}}=\boldsymbol{B}(\boldsymbol{q}) \delta \boldsymbol{q}
$$

with

$$
\boldsymbol{B}(\boldsymbol{q})=\left[\begin{array}{c}
\boldsymbol{q}^{T} \boldsymbol{A}_{11} \\
\boldsymbol{q}^{T} \boldsymbol{A}_{22} \\
\boldsymbol{q}^{T} \boldsymbol{A}_{33} \\
2 \boldsymbol{q}^{T} \boldsymbol{A}_{12} \\
2 \boldsymbol{q}^{T} \boldsymbol{A}_{23} \\
2 \boldsymbol{q}^{T} \boldsymbol{A}_{13}
\end{array}\right] .
$$

Then, using Eq. (16), the variation of the local-Cartesian components of $\boldsymbol{E}$ is

$$
\delta \check{\boldsymbol{E}}=\boldsymbol{\Theta} \boldsymbol{B} \delta \boldsymbol{q}
$$

where $\boldsymbol{B} \equiv \boldsymbol{B}(\boldsymbol{q})$ 


\subsection{External forces and couples}

The (external) virtual work produced by the displacement $\delta \boldsymbol{u}$ or, equivalently, the nodal variations $\delta \boldsymbol{q}$, is

$$
\mathcal{W}^{\mathrm{ext}}=\boldsymbol{F}^{\mathrm{ext}} \cdot \delta \boldsymbol{q}
$$

introducing $\boldsymbol{F}^{\text {ext }}$ as the vector of external forces and couples lumped at the nodes.

\subsection{Elimination of the drilling degree of freedom}

Following a common practice in the formulation of MITCn elements, we choose to eliminate the drilling degree of freedom, that associated to the rotation of the shell around the director. But differing from the classical papers on MITCn, where an additive scheme is used to update the director, recourse is made to the multiplicative scheme proposed by Simo et al.[11], which avoids singularities for large rotations and guarantees the inextensibility of the director. Using such a scheme, the variation of $\boldsymbol{t}$ at node $i$ is expressed as

$$
\delta \boldsymbol{t}_{i}=\tilde{\boldsymbol{\lambda}}_{i} \delta \boldsymbol{t}_{i}^{*} \quad(\text { no summation over } i)
$$

where $\delta \boldsymbol{t}_{i}^{*}$ is a vector in the plane $\{\boldsymbol{i}, \boldsymbol{j}\}$ of the fixed global Cartesian frame $\{\boldsymbol{i}, \boldsymbol{j}, \boldsymbol{k}\}$, and $\tilde{\boldsymbol{\lambda}}_{i}$ is the $2 \times 3$ matrix made of the first two rows of the orthogonal matrix $\boldsymbol{\lambda}_{i}$ from the transformation

$$
\boldsymbol{t}_{i}=\boldsymbol{\lambda}_{i} \boldsymbol{k}
$$

Eq. (27) shows that only two degrees of freedom are needed to update the nodal director, this way eliminating the drilling degree of freedom, making 
the current formulation have five degrees of freedom per node. Consequently, $\delta \boldsymbol{q}$ is replaced by

$$
\delta \boldsymbol{q}=\underbrace{\left[\begin{array}{ccccc}
\boldsymbol{I}_{3 \times 3} & \boldsymbol{0}_{3 \times 2} & \cdots & \boldsymbol{0}_{3 \times 3} & \boldsymbol{0}_{3 \times 2} \\
\boldsymbol{0}_{3 \times 3} & \tilde{\boldsymbol{\lambda}}_{1} & \cdots & \boldsymbol{0}_{3 \times 3} & \boldsymbol{0}_{3 \times 2} \\
\vdots & \vdots & \ddots & \vdots & \vdots \\
\boldsymbol{0}_{3 \times 3} & \boldsymbol{0}_{3 \times 2} & \cdots & \boldsymbol{I}_{3 \times 3} & \boldsymbol{0}_{3 \times 2} \\
\boldsymbol{0}_{3 \times 3} & \boldsymbol{0}_{3 \times 2} & \cdots & \boldsymbol{0}_{3 \times 3} & \tilde{\boldsymbol{\lambda}}_{N}
\end{array}\right]}_{\boldsymbol{\Lambda}} \underbrace{\left[\begin{array}{c}
\delta \overline{\boldsymbol{x}}_{1} \\
\delta \boldsymbol{t}_{1}^{*} \\
\vdots \\
\delta \overline{\boldsymbol{x}}_{N} \\
\delta \boldsymbol{t}_{N}^{*}
\end{array}\right]}_{\delta \boldsymbol{q}^{*}}
$$

where $\boldsymbol{0}_{i \times j}$ denotes the $i \times j$ zero matrix.

\subsection{Discrete equilibrium equations for the degenerate solid shell FEM}

By replacing $\delta \boldsymbol{q}$ by $\delta \boldsymbol{q}^{*}$, taking into account that $\delta \boldsymbol{q}^{*}$ is arbitrary, and introducing the strain and stress measures in local Cartesian coordinates, the PVW gives rise to the discrete, nonlinear system of algebraic equations that governs the equilibrium of the degenerate solid shell FEM:

$$
\boldsymbol{R}^{*}=\boldsymbol{F}^{\mathrm{int} *}-\boldsymbol{F}^{\mathrm{ext} *}=\boldsymbol{O},
$$

where

$$
\begin{aligned}
& \boldsymbol{F}^{\mathrm{ext} *}=\boldsymbol{\Lambda}^{T} \boldsymbol{F}^{\mathrm{ext}}, \\
& \boldsymbol{F}^{\mathrm{int} *}=\boldsymbol{\Lambda}^{T} \underbrace{\int_{\mathcal{B}^{0}} \boldsymbol{B}^{T} \boldsymbol{\Theta}^{T} \check{\boldsymbol{S}} \mathrm{d} V}_{\boldsymbol{F}^{\mathrm{int}}},
\end{aligned}
$$

where the vector $\boldsymbol{F}^{\text {int }}$ of nodal internal forces and couples is introduced, with $\check{\boldsymbol{S}}$ denoting the vector made of the components $S_{i j}$ of the stress tensor $\boldsymbol{S}$ with respect to the local-Cartesian frame $\left\{\boldsymbol{e}_{i}\right\}$ ordered according to Voigt 
notation:

$$
\check{\boldsymbol{S}}=\left[\begin{array}{llllll}
S_{11} & S_{22} & S_{33} & S_{12} & S_{23} & S_{13}
\end{array}\right]^{T}
$$

\section{Inverse finite element analysis}

In inverse finite element analysis, the loaded configuration $B$ as well as the external loads responsible for deforming the shell from $B^{0}$ to $B$ are assumed to be known. In our previous papers [3, 4], we chose to formulate the equilibrium equation over the known configuration $B$, using Eulerian stress and strain measures. In the present paper, we adopt a different approach, assuming that both FEM and IFEM have the same governing equations, that given by the discrete equilibrium equation, Eq. (30), differing only in the fact that the knowns and unknowns are interchanged. Let us explicitly state how the terms involved in the governing equation, Eq. (30), depend on $\boldsymbol{q}$ and/or $\boldsymbol{Q}$. Considering $\boldsymbol{F}^{\text {int* }}$, we have

$$
\begin{aligned}
\boldsymbol{F}^{\mathrm{int} *}(\boldsymbol{Q}, \boldsymbol{q}) & =\boldsymbol{\Lambda}^{T}(\boldsymbol{q}) \int_{\mathcal{B}^{0}(\boldsymbol{Q})} \boldsymbol{B}^{T}(\boldsymbol{q}) \boldsymbol{\Theta}^{T}(\boldsymbol{Q}) \check{\boldsymbol{S}}(\boldsymbol{Q}, \boldsymbol{q}) \mathrm{d} V(\boldsymbol{Q}) \\
& =\boldsymbol{\Lambda}^{T}(\boldsymbol{q}) \int_{\mathcal{B}(\boldsymbol{q})} \boldsymbol{B}^{T}(\boldsymbol{q}) \boldsymbol{\Theta}^{T}(\boldsymbol{Q}) \check{\boldsymbol{S}}(\boldsymbol{Q}, \boldsymbol{q})[J(\boldsymbol{Q}, \boldsymbol{q})]^{-1} \mathrm{~d} v(\boldsymbol{q}),
\end{aligned}
$$

where the last equality was obtained by a simple change of the integration domain, with $J$ the Jacobian determinant of the transformation from $\mathcal{B}^{0}$ to $\mathcal{B}$, given by

$$
J=\frac{\mathrm{d} v}{\mathrm{~d} V}=\frac{\left(\boldsymbol{g}_{1} \times \boldsymbol{g}_{2}\right) \cdot \boldsymbol{g}_{3}}{\left(\boldsymbol{G}_{1} \times \boldsymbol{G}_{2}\right) \cdot \boldsymbol{G}_{3}}
$$

In the FEM, $\boldsymbol{F}^{\text {int* }}$ depends on the unknown $\boldsymbol{q}$ via $\boldsymbol{\Lambda}, \boldsymbol{B}$ and $\check{\boldsymbol{S}}$, while in the IFEM, it depends on the unknown $\boldsymbol{Q}$ via $\boldsymbol{\Theta}, J$, and $\check{\boldsymbol{S}}$. 
Concerning the external loads, they generally depend on both the deformed and undeformed configurations:

$$
\boldsymbol{F}^{\mathrm{ext} *}(\boldsymbol{Q}, \boldsymbol{q})=\boldsymbol{\Lambda}^{T}(\boldsymbol{q}) \boldsymbol{F}^{\mathrm{ext}}(\boldsymbol{Q}, \boldsymbol{q}) .
$$

In the case of a pressure load, that is, a configuration-dependent load, $\boldsymbol{F}^{\text {ext* }}$ is constant for IFEM. In contrast, for a dead load, $\boldsymbol{F}^{\text {ext* }}$ is a nonlinear function of $\boldsymbol{Q}$.

\subsection{Solution of the nonlinear equilibrium equation in IFEM}

Let us rewrite the equilibrium equations (30) as

$$
\boldsymbol{R}^{*}(\boldsymbol{Q})=\boldsymbol{F}^{\mathrm{int} *}(\boldsymbol{Q}, \boldsymbol{q})-\boldsymbol{F}^{\mathrm{ext} *}(\boldsymbol{Q}, \boldsymbol{q}) .
$$

When specifically applied to the IFEM, the system of equations (37) have $\boldsymbol{q}$ as known and $\boldsymbol{Q}$ as unknown. This is a nonlinear system that will be solved using the Newton-Raphson scheme: once $\boldsymbol{Q}^{(k)}$ (that is $\boldsymbol{Q}$ at iteration $k$ ) is known, $\boldsymbol{Q}$ is updated by solving the following linear equation for $\Delta \boldsymbol{Q}^{*}$ :

$$
\boldsymbol{R}^{*}\left(\boldsymbol{Q}^{(k+1)}\right)=\boldsymbol{R}^{*}\left(\boldsymbol{Q}^{(k)}\right)+\boldsymbol{K}^{*}\left(\boldsymbol{Q}^{(k)}\right) \Delta \boldsymbol{Q}^{*}=\boldsymbol{O},
$$

where $\boldsymbol{K}^{*}$ is the tangent stiffness matrix

$$
\boldsymbol{K}^{*}=\frac{\partial \boldsymbol{R}}{\partial \boldsymbol{Q}^{*}}
$$

and

$$
\Delta \boldsymbol{Q}^{*}=\left[\begin{array}{c}
\Delta \overline{\boldsymbol{X}}_{1} \\
\Delta \boldsymbol{T}_{1}^{*} \\
\vdots \\
\Delta \overline{\boldsymbol{X}}_{N} \\
\Delta \boldsymbol{T}_{N}^{*}
\end{array}\right]
$$


After solving the linear system (38), the position of the node $i$ at the undeformed midsurface is straightforwardly updated:

$$
\overline{\boldsymbol{X}}_{i}^{(k+1)}=\overline{\boldsymbol{X}}_{i}^{(k)}+\Delta \overline{\boldsymbol{X}}_{i}
$$

\subsubsection{Update of the material director vector}

The iterative update of the nodal material director $\boldsymbol{T}_{i}$ requires a special treatment for two reasons: first, to preserve its unit length, and second, to transform the 2D-solution $\Delta \boldsymbol{T}_{i}^{*}$. We proceed here in a way identical to that proposed by Simo et al. [10] for the "direct" shell FEM.

Given the initial guess $\boldsymbol{T}_{i}^{(0)}$, we compute the rotation matrix

$$
\boldsymbol{\chi}_{i}^{(0)}=\left(\boldsymbol{k} \cdot \boldsymbol{T}_{i}^{(0)}\right) \boldsymbol{I}_{3 \times 3}+\widehat{\boldsymbol{k} \times \boldsymbol{T}_{i}^{(0)}}+\frac{\left(\boldsymbol{k} \times \boldsymbol{T}_{i}^{(0)}\right) \otimes\left(\boldsymbol{k} \times \boldsymbol{T}_{i}^{(0)}\right)}{1+\boldsymbol{k} \cdot \boldsymbol{T}_{i}^{(0)}},
$$

where $\widehat{\boldsymbol{v}}$ is the skew-symmetric matrix whose axial vector is $\boldsymbol{v}$. Usually,

$\boldsymbol{T}_{i}^{(0)} \equiv \boldsymbol{t}_{i}$ is adopted as the initial guess. In this case, $\boldsymbol{\chi}_{i}^{(0)} \equiv \boldsymbol{\lambda}_{i}$ is the orthogonal matrix of Eq. (28).

Then, once the director $\boldsymbol{T}^{(k)}$ and the rotation matrix $\boldsymbol{\chi}_{i}^{(k)}$ are known for a given iteration $k, \boldsymbol{T}_{i}$ and $\boldsymbol{\chi}_{i}$ are successively updated according to the next steps:

1. Update of the director:

$$
\boldsymbol{T}_{i}^{(k+1)}=\cos \left\|\Delta \boldsymbol{T}_{i}\right\| \boldsymbol{T}_{i}^{(k)}+\frac{\sin \left\|\Delta \boldsymbol{T}_{i}\right\|}{\left\|\Delta \boldsymbol{T}_{i}\right\|} \Delta \boldsymbol{T}_{i},
$$

with

$$
\Delta \boldsymbol{T}_{i}=\left[\tilde{\boldsymbol{\chi}}_{i}^{(k)}\right]^{T} \Delta \boldsymbol{T}_{i}^{*},
$$

where $\tilde{\boldsymbol{\chi}}_{i}^{(k)}$ is the $2 \times 3$ matrix made of the first two rows of $\boldsymbol{\chi}_{i}^{(k)}$. 
2. Update of the rotation matrix:

$$
\boldsymbol{\chi}_{i}^{(k+1)}=\Delta \boldsymbol{\chi}_{i} \boldsymbol{\chi}_{i}^{(k)}
$$

with

$$
\begin{aligned}
\Delta \boldsymbol{\chi}_{i}=\cos \left\|\Delta \boldsymbol{T}_{i}\right\| \boldsymbol{I}_{3 \times 3}+\frac{\sin \left\|\Delta \boldsymbol{T}_{i}\right\|}{\left\|\Delta \boldsymbol{T}_{i}\right\|} \boldsymbol{T}_{i}^{\left(\widehat{k)} \times \Delta \boldsymbol{T}_{i}\right.} \\
+\frac{1-\cos \left\|\Delta \boldsymbol{T}_{i}\right\|}{\left\|\Delta \boldsymbol{T}_{i}\right\|^{2}}\left(\boldsymbol{T}_{i}^{(k)} \times \Delta \boldsymbol{T}_{i}\right) \otimes\left(\boldsymbol{T}_{i}^{(k)} \times \Delta \boldsymbol{T}_{i}\right) .
\end{aligned}
$$

\subsection{Computation of the tangent stiffness matrix}

The tangent matrix $\boldsymbol{K}^{*}$ is made of contributions from the internal and external forces:

$$
\boldsymbol{K}^{*}=\frac{\partial \boldsymbol{F}^{\mathrm{int} *}}{\partial \boldsymbol{Q}^{*}}+\frac{\partial \boldsymbol{F}^{\mathrm{ext} *}}{\partial \boldsymbol{Q}^{*}}=\boldsymbol{K}^{\mathrm{int} *}+\boldsymbol{K}^{\mathrm{ext} *}
$$

The term $\boldsymbol{K}^{\text {ext* }}$ appears only if the external loads depend on the initial configuration, like dead loads. In any case, it will not receive further consideration here.

The contribution of the internal forces given by Eq. (34) can be expressed as

$$
\boldsymbol{K}^{\text {int* }}=\boldsymbol{\Lambda}^{T}\left(\boldsymbol{K}^{\text {mat }}+\boldsymbol{K}^{\text {geo }}\right) \frac{\mathrm{d} \boldsymbol{Q}}{\mathrm{d} \boldsymbol{Q}^{*}},
$$

with

$$
\begin{aligned}
\boldsymbol{K}^{\text {mat }} & =\int_{\mathcal{B}} \boldsymbol{B}^{T} \boldsymbol{\Theta}^{T} \check{\boldsymbol{C}} \frac{\partial \check{\boldsymbol{E}}}{\partial \boldsymbol{Q}} J^{-1} \mathrm{~d} v \\
\boldsymbol{K}^{\text {geo }} & =\left.\int_{\mathcal{B}} \boldsymbol{B}^{T} \frac{\partial\left(\boldsymbol{\Theta}^{T} \boldsymbol{v}\right)}{\partial \boldsymbol{Q}}\right|_{\boldsymbol{v}=\check{\boldsymbol{S}}} J^{-1} \mathrm{~d} v-\int_{\mathcal{B}} \boldsymbol{B}^{T} \boldsymbol{\Theta}^{T} \check{\boldsymbol{S}} J^{-2} \frac{\mathrm{d} J}{\mathrm{~d} \boldsymbol{Q}} \mathrm{d} v .
\end{aligned}
$$




\subsubsection{Computation of $\boldsymbol{K}^{\text {mat }}$}

Two matrices remain undefined in Eq. (49) for $\boldsymbol{K}^{\text {mat }}$. The first one is

$$
\check{\boldsymbol{C}}=\frac{\partial \check{\boldsymbol{S}}}{\partial \check{\boldsymbol{E}}}
$$

which is the matrix (in Voigt notation) containing the tangent moduli $C_{i j k l}=$ $\partial S_{i j} / \partial E_{k l}$ referred to the local-Cartesian base $\left\{\boldsymbol{e}_{i}\right\}$, which are given by the material properties.

The second one is $\partial \check{\boldsymbol{E}} / \partial \boldsymbol{Q}$ that, given $\check{\boldsymbol{E}}$ by Eq. (16), is defined as

$$
\frac{\partial \check{\boldsymbol{E}}}{\partial \boldsymbol{Q}}=\frac{\partial}{\partial \boldsymbol{Q}}\left(\boldsymbol{\Theta} \check{\boldsymbol{E}}^{\mathrm{cov}}\right)=\left.\frac{\partial(\boldsymbol{\Theta} \boldsymbol{v})}{\partial \boldsymbol{Q}}\right|_{\boldsymbol{v}=\check{\boldsymbol{E}}^{\mathrm{cov}}}+\boldsymbol{\Theta} \frac{\mathrm{d} \check{\boldsymbol{E}}^{\mathrm{cov}}}{\mathrm{d} \boldsymbol{Q}} .
$$

The first term in the r.h.s. of Eq. (52) is the matrix whose $i j$ entry is

$$
\left[\frac{\partial(\boldsymbol{\Theta} \boldsymbol{v})}{\partial \boldsymbol{Q}}\right]_{i j}=\frac{\partial \Theta_{i k}}{\partial Q_{j}} v_{k} .
$$

Now, it remains to compute $\partial \Theta_{i k} / \partial Q_{j}$. Given $\boldsymbol{\Theta}$ by Eq. (19), its derivative with respect to $Q_{j}$ is completely determined by the knowledge of

$$
\frac{\mathrm{d} \theta_{i}^{\alpha}}{\mathrm{d} Q_{j}}=\boldsymbol{e}_{i} \cdot \frac{\mathrm{d} \boldsymbol{G}^{\alpha}}{\mathrm{d} Q_{j}} .
$$

Since $\boldsymbol{G}^{\alpha} \cdot \boldsymbol{G}_{\beta}=\delta_{\beta}^{\alpha}$, we have

$$
\frac{\partial G_{i}^{\alpha}}{\partial Q_{j}}=-G_{k}^{\alpha} \frac{\partial G_{\beta_{k}}}{\partial Q_{j}} G_{i}^{\beta},
$$

where it remains to determine $\partial G_{\beta_{k}} / \partial Q_{j}$. Taking into account that $\boldsymbol{G}_{\beta}=$ $\left(\partial \boldsymbol{\Phi} / \partial \xi_{\beta}\right) \boldsymbol{Q}$ when $\boldsymbol{X}$ is interpolated according to Eq. (3), $\partial G_{\beta_{k}} / \partial Q_{j}$ is the $k j$ entry of the matrix

$$
\frac{\partial \boldsymbol{G}_{\beta}}{\partial \boldsymbol{Q}}=\frac{\partial \boldsymbol{\Phi}}{\partial \xi_{\beta}} .
$$


Regarding the second term in the r.h.s. of Eq. (52), it remains to compute the matrix $\partial \boldsymbol{E}^{\mathrm{cov}} / \partial \boldsymbol{Q}$. Given the covariant strain components $E_{\alpha \beta}^{\text {cov }}$ by Eq. (8), we have

$$
\frac{\partial \check{\boldsymbol{E}}^{\mathrm{cov}}}{\partial \boldsymbol{Q}}=-\boldsymbol{B}^{0},
$$

with $\boldsymbol{B}^{0} \equiv \boldsymbol{B}(\boldsymbol{Q})$ defined by Eq. (24).

Finally, $\boldsymbol{K}^{\text {mat }}$ takes the form

$$
\boldsymbol{K}^{\mathrm{mat}}=\left.\int_{\mathcal{B}} \boldsymbol{B}^{T} \boldsymbol{\Theta}^{T} \check{\boldsymbol{C}} \frac{\partial(\boldsymbol{\Theta} \boldsymbol{v})}{\partial \boldsymbol{Q}}\right|_{\check{\boldsymbol{E}}^{\text {cov }}} J^{-1} \mathrm{~d} v-\int_{\mathcal{B}} \boldsymbol{B}^{T} \boldsymbol{\Theta}^{T} \check{\boldsymbol{C}} \boldsymbol{\Theta} \boldsymbol{B}^{0} J^{-1} \mathrm{~d} v .
$$

\subsubsection{Computation of $\boldsymbol{K}^{\text {geo }}$}

Regarding the first term of $\boldsymbol{K}^{\text {geo }}$, Eq. (50), it only remains to determine

$$
\left[\frac{\partial\left(\boldsymbol{\Theta}^{T} \boldsymbol{v}\right)}{\partial \boldsymbol{Q}}\right]_{i j}=\frac{\partial \Theta_{k i}}{\partial Q_{j}} v_{k}
$$

where $\partial \Theta_{k i} / \partial Q_{j}$ can be expressed in terms of $\partial \theta_{i}^{\alpha} / \partial Q_{j}$, Eq. (54).

Regarding the second term of Eq. (50), we need to compute $\partial J / \partial Q_{j}$. Invoking Eq. (35), we have

$$
\begin{aligned}
& \frac{\partial J}{\partial Q_{j}}=-\frac{J}{\left(\boldsymbol{G}_{1} \times \boldsymbol{G}_{2}\right) \cdot \boldsymbol{G}_{3}} \times \\
& \varepsilon_{p q r}\left(\frac{\partial G_{1_{q}}}{\partial Q_{j}} G_{2_{r}} G_{3_{p}}+G_{1_{q}} \frac{\partial G_{2_{r}}}{\partial Q_{j}} G_{3_{p}}+G_{1_{q}} G_{2_{r}} \frac{\partial G_{3_{p}}}{\partial Q_{j}}\right),
\end{aligned}
$$

where $\epsilon_{p q r}$ is the Levi-Civita or permutation symbol, and $\partial G_{\alpha_{i}} / \partial Q_{j}$ is defined by Eq. (56). 


\subsubsection{Computation of $\partial \boldsymbol{Q} / \partial \boldsymbol{Q}^{*}$}

Once $\boldsymbol{K}^{\text {mat }}$ and $\boldsymbol{K}^{\text {geo }}$ are completely determined, the complete determination of the tangent stiffness matrix $\boldsymbol{K}^{\text {int* }}$, Eq. (48), requires computing

$$
\frac{\partial \boldsymbol{Q}}{\partial \boldsymbol{Q}^{*}}=\left[\begin{array}{ccccc}
\boldsymbol{I}_{3 \times 3} & \boldsymbol{0}_{3 \times 2} & \cdots & \boldsymbol{0}_{3 \times 3} & \boldsymbol{O}_{3 \times 2} \\
\boldsymbol{0}_{3 \times 3} & \partial \boldsymbol{T}_{1} / \partial \Delta \boldsymbol{T}_{1}^{*} & \cdots & \boldsymbol{O}_{3 \times 3} & \boldsymbol{0}_{3 \times 2} \\
\vdots & \vdots & \ddots & \vdots & \vdots \\
\boldsymbol{0}_{3 \times 3} & \boldsymbol{0}_{3 \times 2} & \cdots & \boldsymbol{I}_{3 \times 3} & \boldsymbol{0}_{3 \times 2} \\
\boldsymbol{O}_{3 \times 3} & \boldsymbol{O}_{3 \times 2} & \cdots & \boldsymbol{0}_{3 \times 3} & \partial \boldsymbol{T}_{N} / \partial \Delta \boldsymbol{T}_{N}^{*}
\end{array}\right]
$$

where we need to determine $\partial \boldsymbol{T}_{i} / \partial \Delta \boldsymbol{T}_{i}^{*}$ by taking into account the update procedure described in Section 3.1.1. By differentiating the updated $\boldsymbol{T}_{i}$ given by Eq. (43) with respect to the nodal increment $\Delta \boldsymbol{T}_{i}^{*}$, we obtain

$$
\begin{gathered}
\frac{\partial \boldsymbol{T}_{i}^{(k+1)}}{\partial \Delta \boldsymbol{T}_{i}^{*}}=\frac{\partial \boldsymbol{T}_{i}^{(k+1)}}{\partial \Delta \boldsymbol{T}_{i}} \frac{\partial \Delta \boldsymbol{T}_{i}}{\partial \Delta \boldsymbol{T}_{i}^{*}}=\left[-\frac{\cos \left\|\Delta \boldsymbol{T}_{i}\right\|}{\left\|\Delta \boldsymbol{T}_{i}\right\|} \boldsymbol{T}_{i}^{(k)} \otimes \Delta \boldsymbol{T}_{i}+\right. \\
\left(\frac{\cos \left\|\Delta \boldsymbol{T}_{i}\right\|}{\left\|\Delta \boldsymbol{T}_{i}\right\|^{2}}-\frac{\sin \left\|\Delta \boldsymbol{T}_{i}\right\|}{\left\|\Delta \boldsymbol{T}_{i}\right\|^{3}}\right) \\
\left.\frac{\sin \| \Delta \boldsymbol{T}_{i} \otimes \Delta \boldsymbol{T}_{i}+}{\left\|\Delta \boldsymbol{T}_{i}\right\|} \boldsymbol{I}_{3 \times 3}\right]\left[\tilde{\boldsymbol{\chi}}_{i}^{(k)}\right]^{T} .
\end{gathered}
$$

\section{Applications}

In this section, three benchmarks are solved, to illustrate how IFEM works in the context of shell analysis. These three problems are based on popular benchmarks for the geometrically non-linear analysis of shells using (direct) FEM [17], serving by the way as a validation of the presented IFEM model.

Next, IFEM will be applied to the design of a compliant microvalve. This real-life application is exemplary of the strength of IFEM for the design of compliant structures to attain a given shape after large elastic deformations. 


\subsection{Bending of a cantilever}

Let us consider the cantilever shown in Fig. 2, with length $L=10$, width $w=1$, and thickness $h=0.1$. When unloaded, it lies in the $x y$-plane. Then, it is deformed by a lifting load uniformly distributed along the end, whose resultant is the force $P=4$ in the $z$-direction. The plate is made of a Saint Venant-Kirchhoff (linear-elastic) material, with Young modulus $E=1.2 \times 10^{6}$ and Poisson ratio $\nu=0$.

This problem, a popular benchmark for (direct) FEM applied to shells under large elastic deformations (see [17] and the references therein), serves the purpose of validating the current IFEM.

First, the problem is solved using the direct FEM. To this end, the undeformed domain is represented by a mesh of $8 \times 1$ MITC4 finite elements, obtaining the solution shown in Fig. 2a. This solution is in very good agreement with that of Sze et al. [17], who used the reduced-integration elements known as S4R from the commercial code ABAQUS.

Second, the FEM-computed position $\boldsymbol{x}_{i}$ and director $\boldsymbol{t}_{i}$ at all the nodes of the mesh for FEM are assumed to define the mesh for the IFEM, made once again of MITC4 elements. The deforming load (with resultant $P$ in the $z$-direction) now is distributed along the free end of the mesh for the IFEM. The solution of the IFEM problem is depicted in Fig. 2b.

The accuracy of IFEM is determined by its ability to recover the given undeformed plane cantilever as the solution, and can be measured in terms of

$$
\begin{array}{r}
\operatorname{error}\left(\boldsymbol{X}_{i}\right)=\left\|\boldsymbol{X}_{i}^{\mathrm{FEM}}-\boldsymbol{X}_{i}^{\mathrm{IFEM}}\right\|, \\
\operatorname{error}\left(\boldsymbol{T}_{i}\right)=\left\|\boldsymbol{T}_{i}^{\mathrm{FEM}}-\boldsymbol{T}_{i}^{\mathrm{IFEM}}\right\|,
\end{array}
$$




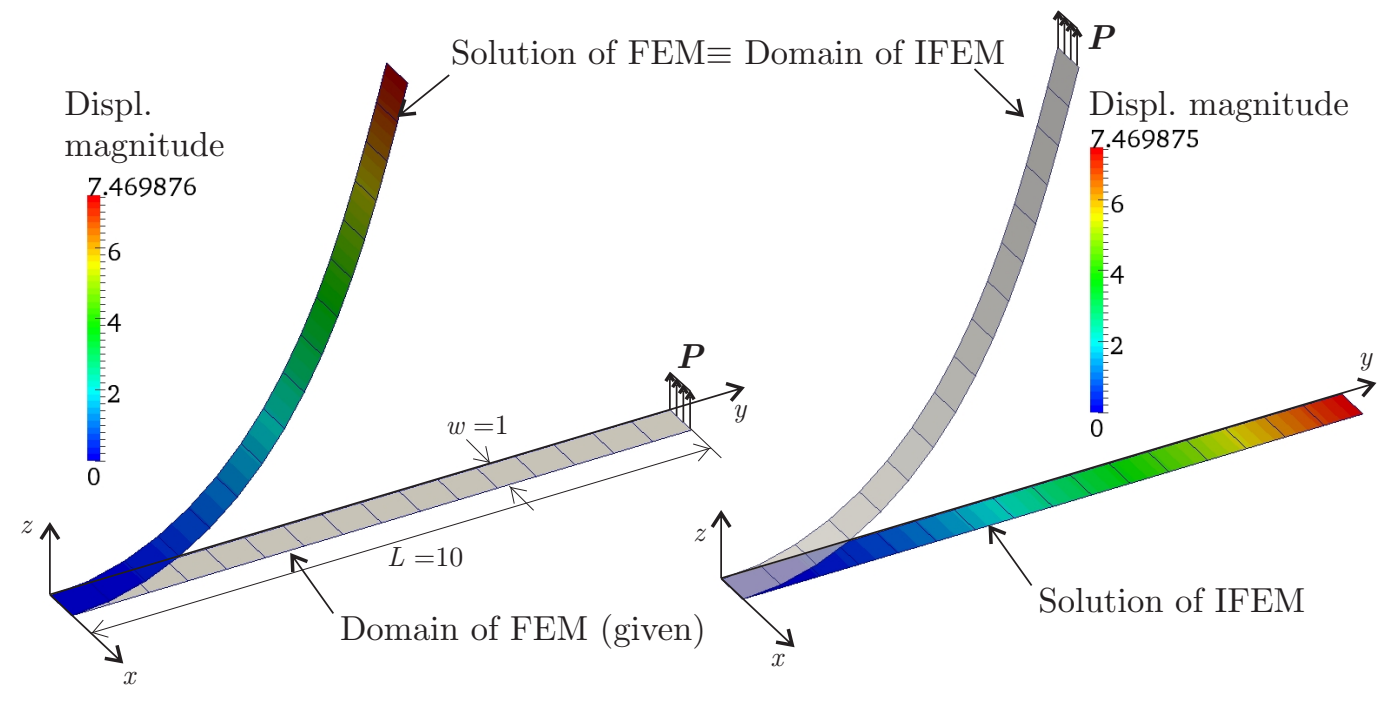
a) FEM problem
b) IFEM problem

Figure 2: Cantilever under large elastic deformation: a) FEM problem with the deformed configuration as solution, b) IFEM problem with the undeformed configuration as solution.

where $(*)_{i}^{\mathrm{FEM}}$ refers to the variable $(*)$ at the node $i$ of the mesh used by FEM, which is known, and $(*)_{i}^{\mathrm{IFEM}}$ refers to the variable $(*)$ at the node $i$ computed as solution of the IFEM.

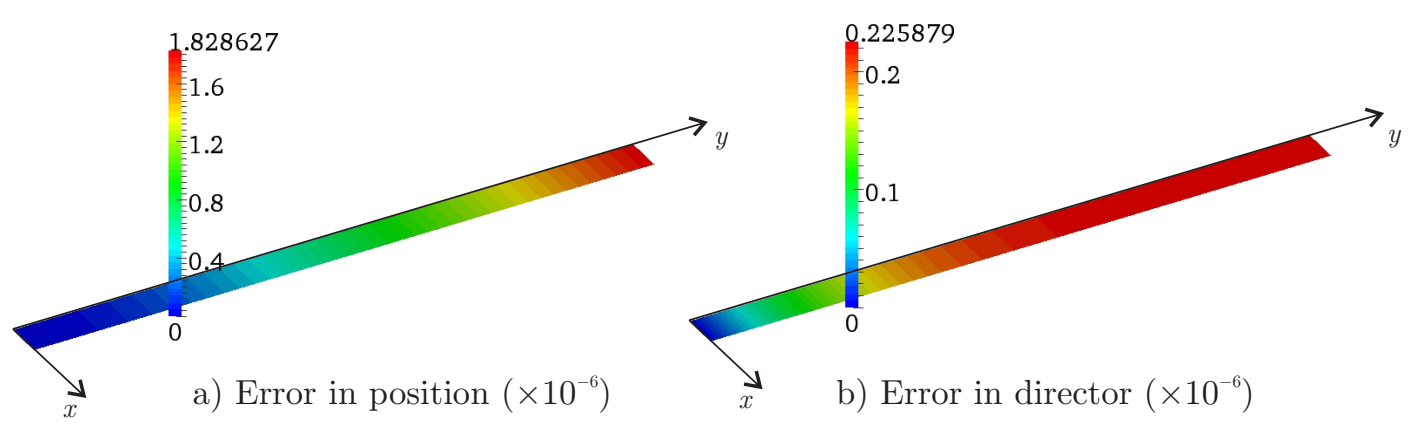

Figure 3: Cantilever under large elastic deformation: accuracy of IFEM.

Fig. 3 proves the remarkabe accuracy of the IFEM in the current ap- 
plication: the maximal error $\left(\boldsymbol{X}_{i}\right)$ is $1.83 \times 10^{-6}$ and occurs at the nodes along the loaded end where the displacement magnitude is 7.47 ; the maximal error $\left(\boldsymbol{T}_{i}\right)$ is $2.26 \times 10^{-7}$ and takes place at the same nodes. Let us remark that both errors depend on the convergence criterion for the solution of the nonlinear equilibrium Eq. (37): in this case, the FEM problem of obtaining the deformed configuration as well as the IFEM problem of recovering the undeformed configuration were solved until $\left\|\boldsymbol{R}^{*}\right\|<10^{-6}$.

Another outstanding quality of the IFEM, already observed in several applications developed in our previous papers $[3,4,6]$, is the fast convergence to the solution of the nonlinear equilibrium Eq. (37).

Actually, when Sze et al. [17] solved the same (direct) problem, they needed to use 78 iterations along 15 load increments, whereas the current IFEM problem was solved using only 14 iterations along 2 load steps.

\subsection{A slit annular plate under a lifting force}

The second benchmark consists of a slit annular plate, with inner radius $r=6$, outer radius $R=10$, and thickness $h=0.03$ clamped at one side of the slit and deformed by a lifting force uniformly distributed along the other side of the slit, with resultant $P=3.2$ and direction given by the $z$-axis (see Fig. 4). When unloaded, the plate lies in the $x y$-plane.

The plate is made of a Saint Venant-Kirchhoff (linear-elastic) material, with Young modulus $E=21 \times 10^{6}$ and Poisson ratio $\nu=0$.

This is also a popular benchmark for geometrically non-linear shells, as in the survey of Sze et al. [17].

First, the problem is solved using the FEM with the plate represented by a mesh of $10 \times 80$ MITC4 finite elements. The results are shown in Fig. 4a, 
and are very close to those obtained by Sze et al. [17] using S4R elements from ABAQUS.

Then, the FEM-computed deformed mesh is adopted as the mesh for the IFEM, as shown in Fig. 4b. The load that caused the deformation (that whose resultant is $P$ and whose direction is the $z$-axis) is distributed along the free end of the mesh for the IFEM.

The IFEM solution is shown in Fig. 4b. The accuracy of the IFEM is highlighted in Fig. 5: the maximal error $\left(\boldsymbol{X}_{i}\right)=9.27 \times 10^{-6}$ is negligible compared to the maximal displacement magnitude (19.33), while the maximal $\operatorname{error}\left(\boldsymbol{T}_{i}\right)=0.85 \times 10^{-6}$ is negligible compared to unity. Both errors have the same order of magnitude as the tolerance for the Newton-Raphson solution of Eq. (37), adopted as $\left\|\boldsymbol{R}^{*}\right\|<10^{-6}$ in this case.

Concerning the performance of the solver of the nonlinear equilibrium equation, when Sze et al. [17] solved the current FEM problem, 347 iterations along 67 load increments were required. Here, the IFEM problem was solved using only 15 iterations along 2 load steps.

\subsection{Cylinder under pulling-out forces}

The third benchmark consists of the pulling-out of a shell that, before loading, is a cylinder with radius $R=4.953$, length $L=10.35$, and thickness $h=0.094$, as shown in Fig. 6a. The cylinder has open ends and is pulled out by two opposite radial forces of magnitude $P=2 \times 10^{5}$ concentrated at diametrically opposed points. This shell is made of a Saint Venant-Kirchhoff (linear-elastic) material, with Young modulus $E=10.5 \times 10^{6}$ and Poisson ratio $\nu=0.3125$.

First, the problem is solved using the FEM. Taking advantage of the 


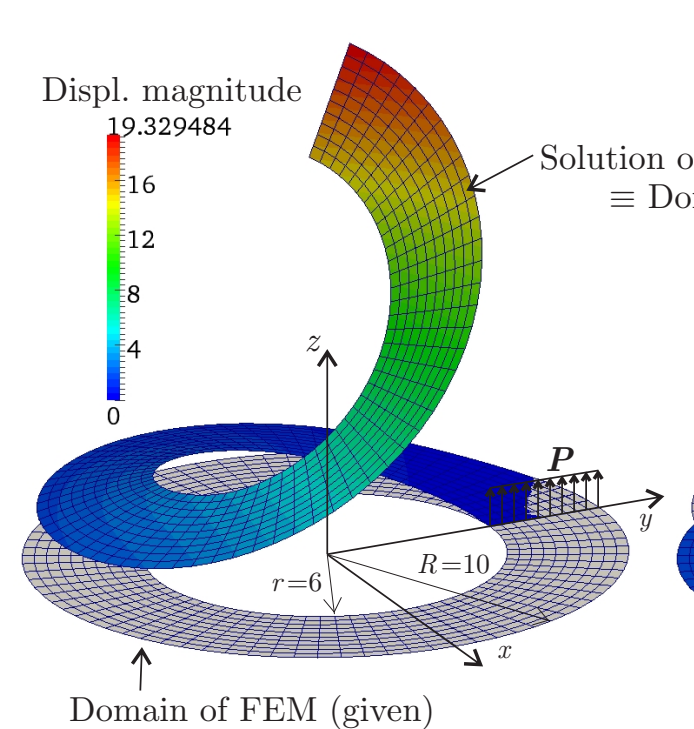

a) FEM problem

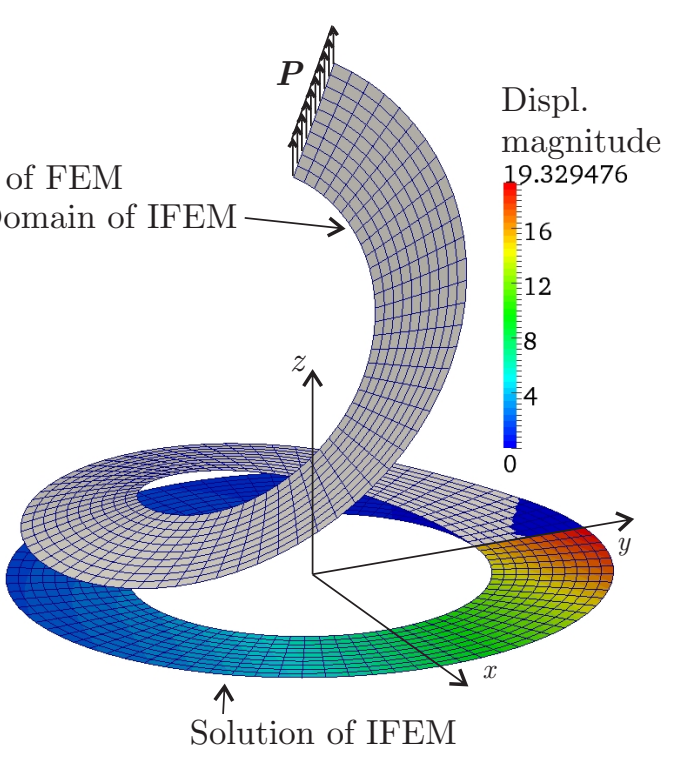

b) IFEM problem

Figure 4: Slit annular plate under large elastic deformation: a) FEM problem with deformed configuration as solution; b) IFEM problem solved to recover the given undeformed configuration.

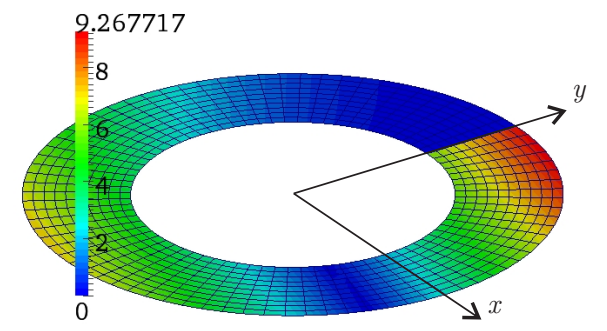

a) Error in position $\left(\times 10^{-6}\right)$

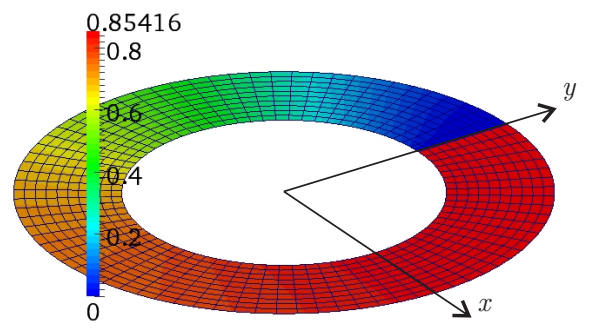

b) Error in director $\left(\times 10^{-6}\right)$

Figure 5: Slit annular plate under large elastic deformation: accuracy of IFEM.

symmetry of the shell with respect to the planes $x z$ and $y z$, only one-fourth of the cylinder is modeled, using 36 (tangential) $\times 48$ (axial) MITC4 elements. The FEM solution is shown in Fig. 4a, and is very close to that obtained by 
Sze et al. [17] using S4R elements from ABAQUS.

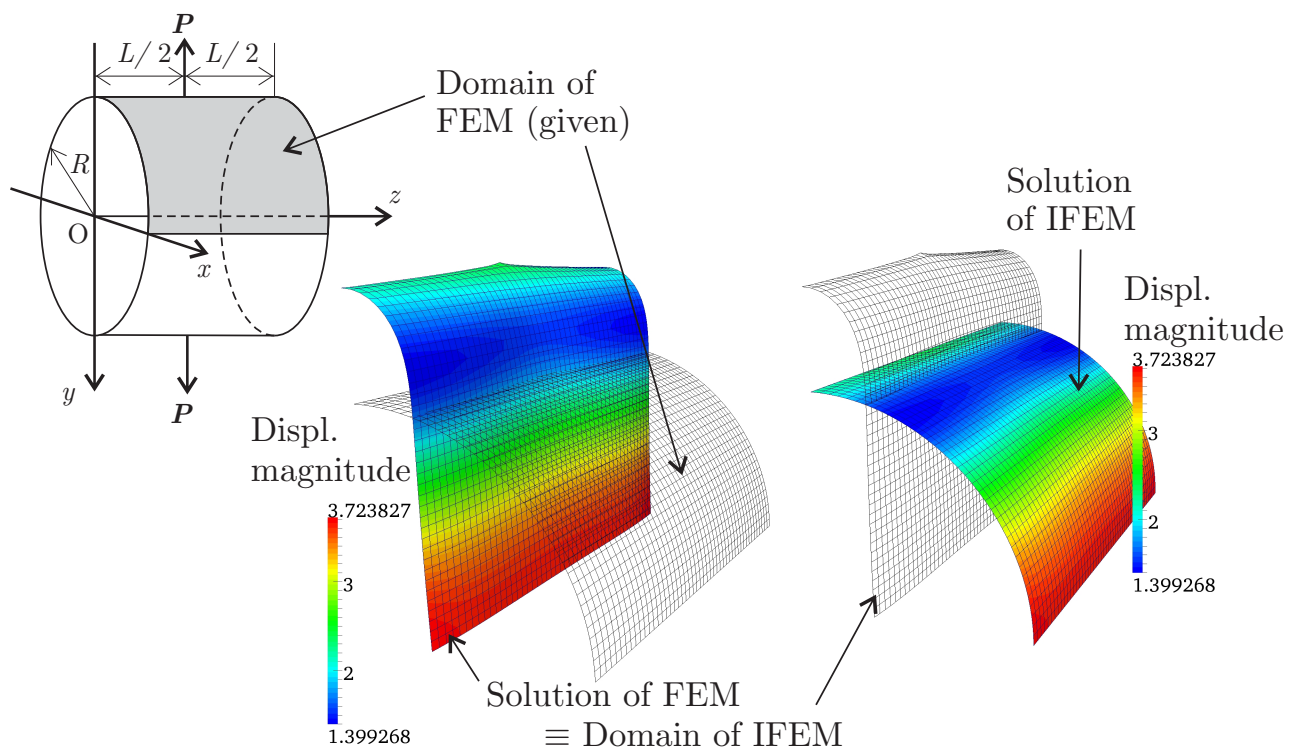
a) FEM problem
b) IFEM problem

Figure 6: Pulled-out cylinder.

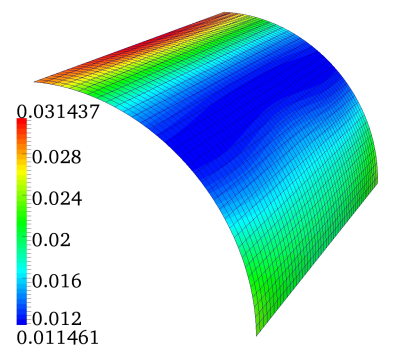

a) Error in position $\left(\times 10^{-6}\right)$

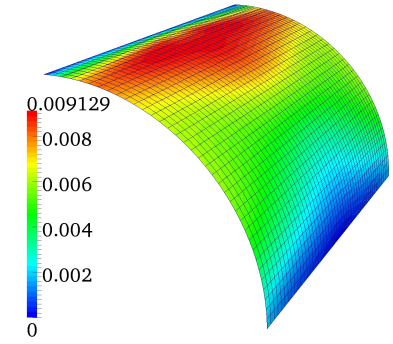

b) Error in director $\left(\times 10^{-6}\right)$

Figure 7: Pulled-out cylinder: accuracy of IFEM.

Second, the FEM-computed deformed mesh is adopted as the mesh for the IFEM, as shown in Fig. 6b. The pulling-out forces have the same magnitude and direction and are applied at the same nodes as those for the FEM. 
The IFEM solution is shown in Fig. 6b. As shown in Fig. 7, the IFEM is able to recover the given undeformed mesh with high accuracy. In this case, the nonlinear equilibrium Eq. (37) was solved until $\left\|\boldsymbol{R}^{*}\right\|<10^{-6}$, requiring 14 iterations along 2 load steps.

\subsection{Design of a passive microvalve}

Now, let us apply the IFEM to a real-life inverse design problem: the design of a passive microvalve whose task is identical to that of the microvalve proposed by Seidemann et al. [18], depicted in Fig. 8. Integrated into a microchannel with thickness $360 \mu \mathrm{m}$ and width $200 \mu \mathrm{m}$, the valve must close the channel when the pressure drop attains a prescribed value $\Delta p$, and bypass a specified flow when the pressure drop vanishes.

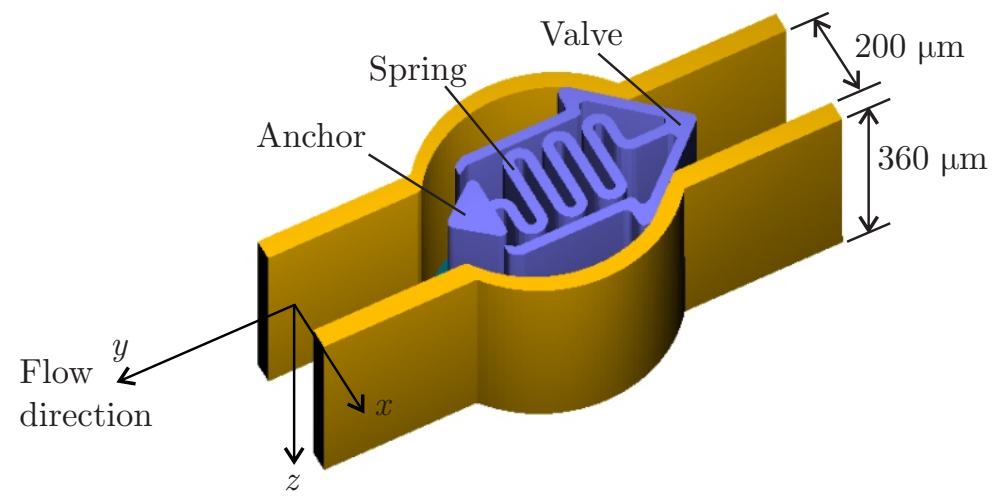

Figure 8: Compliant passive valve to seal a microchannel proposed by Seidemann et al. [18].

Note that the valve in Fig. 8, as originally designed by Seidemann et al. [18], cannot remain centered during deformation because its flexible spring is non-symmetric with respect to the direction of the resultant of the applied pressure. Without information about the pressure drop and the sealing gap, 
it is not possible to assess how critical this defect is in the design of Seidemann et al. [18]. However, Albanesi et al. [6] directly avoided such a defect by replacing the unique non-symmetric spring of the original valve by two springs arranged symmetrically with respect to the axis, as shown in Fig. 9b. This symmetric mechanism, where the springs are compliant beams, is the starting point for the current proposal, shown in Fig. 9b, where the springs behave as shells.

The valve itself is considerably stiffer than the springs, so it is modeled as a rigid body. Further, given the symmetry of the problem, only one spring is modeled, using a mesh of 32850 MITC4 shell elements, each one having sides of approximately $2 \mu \mathrm{m}$. A detail of this fine mesh is shown in Fig. 9c.

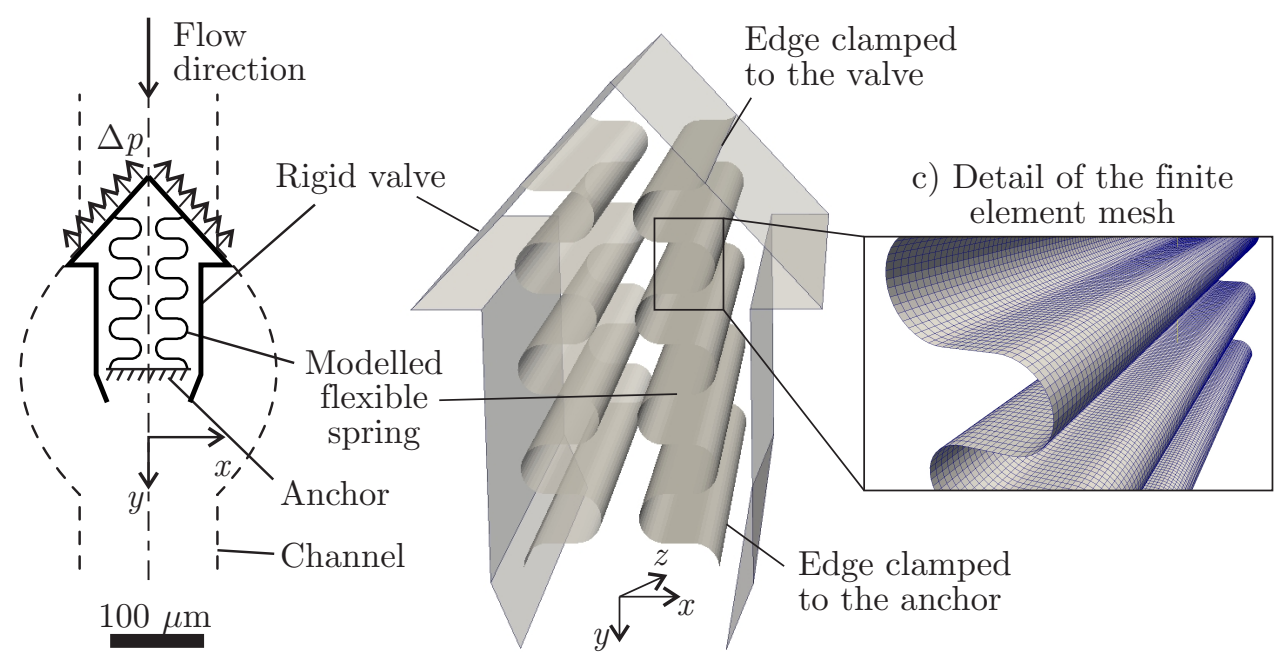
a) Albanesi et al.'s model
b) Current model

Figure 9: Configuration of a compliant passive valve when closed under a given pressure drop $\Delta p$ : a) Albanesi et al.'s model [6], where the springs are made of compliant beams; b) current proposal, where the springs are shells; c) detail of the current finite element mesh. 
The pressure drop $\Delta p$ determining the closure of the valve was not specified by Seidemann et al. [18]. Let us assume $\Delta p=1 \mathrm{kPa}$, which defines the current microvalve as a low-pressure one [21]. The resultant of $\Delta p$ is a force $P=360 \mu$ actuating along the axis of the channel ( $y$-axis in Fig. 9).

Like those of Seidemann et al. [18] and Albanesi et al.[6], the current valve is made of the monomer SU8, which is assumed to be a linear-elastic material with Young modulus $E=3.2 \mathrm{GPa}$ [22], shear modulus $G=1.2 \mathrm{GPa}$ [22], and yield strength from 60 to $73 \mathrm{MPa}$ [23].

There is an additional design requirement that can not be a priori imposed on the IFEM since it involves the valve in its open (i.e., undeformed) condition: a certain sealing gap is needed, depending on the prescribed flow to by-pass. In order to control such a gap for the given geometry of the deformed midsurface, load, and displacement boundary conditions, the thickness of the spring has to be varied. In this case, in order to attain a gap similar to that of the valve of Albanesi et al. [6], the thickness of the spring is set to $h=2 \mu \mathrm{m}$, constant.

The Newton-Raphson solution of the nonlinear equilibrium equation required only one load step and four iterations to attain the convergence criterion $\left\|\boldsymbol{R}^{*}\left(\boldsymbol{Q}^{(4)}\right)\right\|<10^{-5}\left\|\boldsymbol{R}^{*}\left(\boldsymbol{Q}^{(0)}\right)\right\|$, with $\boldsymbol{Q}^{(0)}$ the initial guess with entries $\overline{\boldsymbol{X}}_{i}^{(0)}=\overline{\boldsymbol{x}}_{i}, \boldsymbol{T}_{i}^{(0)}=\boldsymbol{t}_{i}$, where $\overline{\boldsymbol{x}}_{i}$ and $\boldsymbol{t}_{i}$ are the nodal position and director at the node $i$ of the mesh of the given deformed midsurface.

Fig. 10a shows the undeformed configuration computed by IFEM. Note that the maximal displacement takes place at the side where the spring is clamped to the valve, so this is the displacement of the rigid valve itself. The magnitude of such displacement is $24.4 \%$ of the total height of the spring, 
which largely justifies the use of the nonlinear theory of large displacements.

a) Displacement along the axis

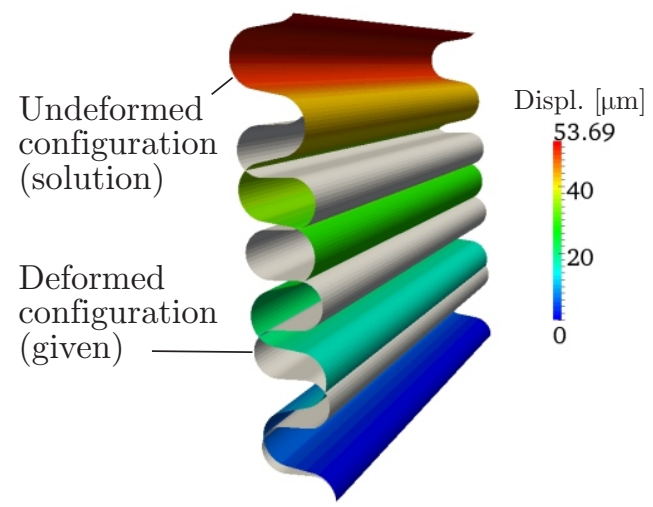

b) Maximal von-Mises stress

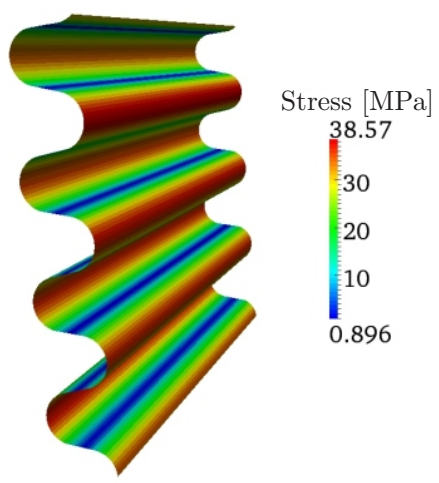

Figure 10: IFEM solution for the compliant passive valve: a) Displacement of the midsurface in the direction of the axis of the channel; b) Maximal von Mises stress across the thickness the shell.

In order to see that the undeformed configuration in Fig. 10a actually constitutes the manufacturing shape of the valve, the feasibility of the IFEM solution has to be evaluated in terms of topological and mechanical tests, as detailed by Albanesi et al. [6]. Concerning the topology, the IFEM may lead to a useless solution containing inter-penetrating elements. As can be seen in Fig. 10, the current solution is free of such defects.

On the other hand, the mechanical tests concern:

1. Validity of the hypothesis of elasticity: assuming the von Mises yield criterion to hold, this is confirmed by Fig. 10b, which shows that the maximal von Mises stress developed throughout the spring, all across its thickness, is considerably lower than the yield strength of SU8 (higher than $60 \mathrm{MPa}[23])$. 
2. Uniqueness of the solution, which is lost when an unstable equilibrium state (or critical point) is met during deformation. In the current case, critical points are not passed through the deformation, which is evident to an experienced designer and can be formally confirmed by using the spectrum test [24].

Having succeeded at all these tests, the IFEM-computed undeformed configuration shown in Fig. 10 represents in fact the manufacturing shape of the springs of the valve, such that this valve exactly closes the channel under the given pressure drop.

\section{Conclusions}

This paper introduced the inverse finite element method (IFEM) for degenerate solid shells. The IFEM is particularly well suited for the inverse design of compliant mechanisms (in this case, shell-like mechanisms) whose task is to attain a desired shape after large elastic deformations. As a good example of an application of the IFEM, the design of a passive valve was undertaken in this paper. Such a design can also be achieved using an optimization technique, where an FEM problem is solved at each iteration. Here, it was achieved by solving only one IFEM problem.

Further, in the light of the current applications, we observe once again (see our previous papers on 3D solids [3] and beams $[4,5,6]$ ) that the solution of the nonlinear equilibrium equation when the undeformed configuration is unknown (the case of the IFEM) takes considerably fewer iterations than the solution of the same equation when the deformed configuration is unknown (the case of the FEM). 
Last but not least, since degenerate solid-shell FEM -unlike the stressresultant shell FEM- makes use of the governing equations from Solid Mechanics, it makes possible to reuse the standard material libraries.

\section{Acknowledgments}

The research for this paper was financially supported by the European Research Council under the European Union's Seventh Framework Programme (FP/2007-2013) / ERC Grant Agreement N. 320815 (ERC Advanced Grant Project "Advanced tools for computational design of engineering materials COMP-DES-MAT"), and the National Scientific and Technical Research Council of Argentina (CONICET) under the grant PIP 2012-2014 GI-1105.

A. E. Albanesi also thanks the National Technological University of Argentina (UTN) for the grant PID ENUTNFE0002146.

\section{References}

[1] S. Govindjee and P. A. Mihalic. Computational methods for inverse finite elastostatics. Comput. Methods Appl. Mech. Engrg., 136:47-57, 1996.

[2] S. Govindjee and P. A. Mihalic. Computational methods for inverse deformations in quasi-incompressible finite elasticity. Int. J. Numer. Meth. Engng., 43:821-838, 1998.

[3] V. Fachinotti, A. Cardona, and P. Jetteur. Finite element modelling of inverse design problems in large deformations anisotropic hyperelasticity. Int. J. Numer. Meth. Engng., 74:894-910, 2008. 
[4] A. E. Albanesi, V. D. Fachinotti, and A. Cardona. Inverse finite element method for large-displacement beams. Int. J. Numer. Meth. Engng., 84: 1166-1182, 2010.

[5] A. E. Albanesi. Inverse design methods for compliant mechanisms. PhD thesis, Faculty of Engineering and Water Sciences, National Littoral University, Santa Fe, Argentina, 2011.

[6] A. E. Albanesi, M. A. Pucheta, and V. D. Fachinotti. A new method to design compliant mechanisms based on the inverse beam finite element model. Mech. Mach. Theory, 65:14-28, 2013.

[7] J. Lu, X. Zhou, and M. L. Raghavan. Computational method of inverse elastostatics for anisotropic hyperelastic solids. Int. J. Numer. Meth. Engng., 69:1239-1261, 2007.

[8] X. Zhou and J. Lu. Inverse formulation for geometrically exact stress resultant shells. Int. J. Numer. Meth. Engng., 74:1278-1302, 2008.

[9] C.-C. Lan and Y.-J. Cheng. Distributed shape optimization of compliant mechanisms using intrinsic functions. In Proceedings of the ASME 2007 International Design Engineering Technical Conferences $\&$ Computer and Engineering Conference, Las Vegas, NV, USA, September 2007.

[10] J. C. Simo, D. D. Fox, and M. S. Rifai. On a stress resultant geometrically exact shell model. Part III: Computational aspects of the nonlinear-theory. Comput. Methods Appl. Mech. Engrg., 79:21-90, 1990.

[11] J. C. Simo and D. D. Fox. On a stress resultant geometrically exact 
shell model. Part I: Formulation and optimal parametrization. Comput. Methods Appl. Mech. Engrg., 72:267-304, 1989.

[12] S. Ahmad, B. M. Irons, and O. C. Zienkiewicz. Analysis of thick and thin shell structures by curved finite elements. Int. J. Numer. Meth. Engng., 2:419-451, 1970.

[13] E. Ramm. A plate/shell element for large deflections and rotations. In K.-J. Bathe, J. T.Oden, and W. Wunderlich, editors, Formulations and Computational Algorithms in Finite Element Analysis: U.S.-Germany Symposium, pages 264-293. Massachusetts Institute of Technology, 1976.

[14] K.-J. Bathe. Finite Element Procedures. Prentice-Hall, 1996.

[15] E. N. Dvorkin and K.-J. Bathe. A continuum mechanics based fournode shell element for general nonlinear analysis. Eng. Comput., 1: 77-88, 1984.

[16] M. L. Bucalem and K.-J. Bathe. Higher-order MITC general shell elements. Int. J. Numer. Meth. Engng., 36:3729-3754, 1993.

[17] K. Y. Sze, X. H. Liu, and S. H. Lo. Popular benchmark problems for geometric nonlinear analysis of shells. Finite Elements in Analysis and Design, 40:1551-1569, 2004.

[18] V. Seidemann, S. Bütefisch, and S. Büttgenbach. Fabrication and investigation of in-plane compliant SU8 structures for MEMS and their application to micro valves and micro grippers. Sensors and Actuators A, 97-98:457-461, 2002. 
[19] M. L. Bucalem and K.-J. Bathe. The Mechanics of Solids and Structures. Hierarchical Modeling and the Finite Element Solution. Computational Fluid and Solid Mechanics. Springer-Verlag, 2011.

[20] D. Chapelle and K.-J. Bathe. The Finite Element Analysis of Shells. Fundamentals. Computational Fluid and Solid Mechanics. SpringerVerlag, second edition, 2011.

[21] A. Pandolfi and M. Ortiz. Improved design of low-pressure fluidic microvalves. J. Micromech. Microeng., 17:1487-1493, 2007.

[22] JAHM Sofware, Inc. Material Property Database MPDB v7.41, 2012.

[23] MicroChem Corp. SU-8 permanent photoresists. Table of properties, 2012.

[24] E. L. Allgower and K. Georg. Introduction to numerical continuation methods. Society for Industrial and Applied Mathematics (SIAM), 2003. 